\section{Is insulin topical gel better than simvastatin topical gel in full thickness wound?}

\author{
Timotius Hansen Arista, ${ }^{1}$ \\ Agus Santoso Budi, ${ }^{1}$ \\ Mirnasari Amirsyah, ${ }^{2}$ \\ Nanda Febry Setyawati ${ }^{3}$
}

${ }^{1}$ Department of Plastic Reconstructive and Aeshetic Surgery, Faculty Medicine Airlangga University, Dr. Soetomo General Hospital, Surabaya, East Java; ${ }^{2}$ Department of General Surgery, Faculty Medicine Syiah Kuala, Dr. Zainoel Abidin General Hospital, Banda Aceh, Aceh; ${ }^{3}$ Department of General Surgery, dr. Soedono General Hospital, Madiun, East Java, Indonesia

\begin{abstract}
Due to issues of limited substances and expensive cost of wound treatment, topical insulin and simvastatin are being developed due to benefits including easy to find and applicable in every health center. Topical insulin can be used in treating diabetic patient's wound. Simvastatin contributed in wound healing process by increasing angiogenesis and lymphangiogenesis. This experimental study is using randomized post test only control design in male Wistar mice, divided in 5 treatment groups with 9 mice each : carboxymethylcellulose sodium gel, short acting insulin topical gel, long acting insulin topical gel, simvastatin topical $2 \%$ gel, and simvastatin 3\% topical gel. A full thickness wound was made randomly and observed in 5 days, followed with wound biopsy and analyzing using histopathological specimen measuring the epithelial thickness, fibroblast proliferation and collagen density from each group. Long acting insulin topical gel has the highest average rate of epithelial proliferation and fibroblast proliferation. In simvastatin 3\% topical gel has the highest level of collagen density level among other groups. This study showed long acting insulin topical gel significantly has better outcome than other groups.
\end{abstract}

\section{Introduction}

Wound is a breakdown in the protective function of the skin; the loss of continuity of skin with or without loss of underlying connective tissue following injury to the skin or underlying tissues/organs caused by surgery, a blow, a cut, chemicals, heat/cold, friction/shear force, pressure or as a result of disease. In treating wound, there are still some limitations due to limited substances, expensive cost, or rural area.

A new modality that being developed is the use of topical insulin and simvastatin. They are rather cheap, easy to find and applicable in every health center. Moreover, topical insulin therapy is hoped to be a modality in treating wound in diabetic patient or patient with normal blood glucose. However, we need to consider the systemic effect of insulin which are hyperglycemia, increasing body weight, insulin edema due to acute salt and water retention, or local effect of insulin injection such as lipohypertrophy or lipoatrophy.5 On the other hand, statin; 3-hydroxy-3-methylglutaryl coenzyme A (HMG-CoA) reductase inhibitor used to decrease plasma cholesterol; has a pleiotropic effect which also decrease the inflammation, atherogenesis, and cardiovascular damage in patient with hypercholesterolemia. ${ }^{1,2}$ Simvastatin is believed has a part in wound healing process by increasing angiogenesis and lymphangiogenesis in wound. ${ }^{1-5}$

Until now, further information about topical insulin and simvastatin are still limited. This study is held to assess the effect of both topicals in epithelial thickness, fibroblast proliferation and collagen density in wound healing process. Epithelial thickness was evaluated using digital visitrak, the proliferation of fibroblast and collagen density were evaluated under microscope.

\section{Materials and Methods}

This experimental study is using randomized post test only control design in male Wistar mice (Rattus norvegicus), which divided in 5 treatment groups: carboxymethylcellulose sodium gel (base of gel), short acting insulin topical gel, long acting insulin topical gel, simvastatin topical $2 \%$ gel, and simvastatin 3\% topical gel. Each group consists of 9 mice, aged 4060 days old and 150-120 grams in weight.

Using anesthesia mixture of 20-30 $\mathrm{mg} / \mathrm{kgBW}$ ketamin and $10 \mathrm{mg} / \mathrm{kgBW}$ xylazine injected intramuscularly, simple random sampling of full thickness wound was created on the back of each mice in day 0 and were observed in day 5 . On the first day, each wound were close treated using topical gel based on their group, covered with transparent dressing and gauze firming the dressing. Then, antibiotic was used to control the infection each mice using intramuscular injection of $100 \mathrm{mg} / \mathrm{kgBW}$ penicillin procaine.

On day 5 , blood withdrawn from each
Correspondence: Timotius Hansen Arista Department of Plastic Reconstructive and Aesthetic Surgery, Faculty of Medicine, Universitas Airlangga, Dr. Soetomo General Hospital, Jl. Prof. Dr. Moestopo no. 47. Tel.: +6231 5020091. Fax: +62315046391. E-mail: timotiushansen@gmail.com

Key words: Wound healing, Simvastatin, Insulin, Topical.

Contributions: The authors contributed equally.

Conflict of interest: The authors have nothing to disclose.

Received for publication: 1 February 2019. Accepted for publication: 13 February 2019.

This work is licensed under a Creative Commons Attribution-NonCommercial 4.0 International License (CC BY-NC 4.0).

(C) Copyright T.H. Arista et al., 2019

Licensee PAGEPress, Italy

Dermatology Reports 2019; 11(s1):8051

doi:10.4081/dr.2019.8051

mice to analyze the blood glucose using glucometer, and the wound were measured using Visitrak Wound Measurement device. Moreover, biopsy from each wound were taken, made into histopathologic specimen which stained using Mallory's Azan (Mallory's Aniline Blue Collagen Stain) and observed under microscope to analyze the epithelial thickness, fibroblast proliferation, and collagen density. Experimental study scheme is showed in Figure 1.

\section{Results}

In epithelial thickness aspects, long acting insulin topical gel has the highest average rate of epithelial proliferation (Figure $2)$. There are significant differences between control group and long acting insulin topical gel group $(\mathrm{p}=0.37)$; and between long acting insulin topical gel group and simvastatin $2 \%$ topical gel $(p=0.24)$.

Long acting insulin topical gel also give the highest average result in fibroblast proliferation after day 5 of study (Figure 3 ). There are significant differences between control with long acting insulin topical gel, short acting insulin topical gel, simvastatin $2 \%$ topical gel, and simvastatin 3\% topical gel $(p=0.00)$. Moreover, there are also a significant difference between long acting insulin topical gel with short acting insulin topical gel, simvastatin $2 \%$ topical gel, and simvastatin $3 \%$ topical gel $(\mathrm{p}=0.00)$. 
According to collagen density level, in simvastatin 3\% topical gel has the highest level among other groups (Figure 4). There are significant differences between control and each group of this study $(\mathrm{p}=0.00)$. However, between simvastatin topical gel and insulin topical gel itself are not significantly different $(\mathrm{p}>0.05)$.

\section{Discussion}

The aim of this study is to analyze the effect of long acting insulin topical gel, short acting insulin topical gel, simvastatin $2 \%$ topical gel, and simvastatin 3\% topical gel in treating a full thickness wound by assessing the epithelial thickness, fibroblast proliferation, and collagen density on the fifth day; which the wound itself is already in proliferation phase.

Insulin topical gel give an effect to keratinocytes proliferation; which by influencing the growth of epithelial cells and microvascular endothelial; and migration from wound edge.4,6 Furthermore, from insulin topical gel itself, there are a significantly difference of epithelial thickness between long acting and short acting topical gel. On the other hand, simvastatin can decrease the level of farnesyl pyrophosphate (FPP) endogen which cause the increasing of keratinocytes migration (in vitro) and epithelial process (ex vivo). Moreover, simvastatin increases neovascularization in ischemic organs by increasing the activation of progenitor cells in endothelial. ${ }^{1,2}$ However, the epithelial thickness in control group and simvastatin $2 \%$ topical gel are not significantly different yet has a significant different with long acting insulin topical gel

Wound healing is started with inflammation phase which induced by increasing platelet activation, neutrophils, and macrophage in 5 or 7 days prior to wound occurred. Proliferation phase will be started as soon as inflammation phase ends, which fibroblast and endothelial proliferation can be seen and give result to increasing level of collagen density. ${ }^{3}$ Collagens are used to make a new connective tissue matrix and contracts the wound. ${ }^{4}$

\section{Conclusions}

From 5 days from this study, we can conclude that long acting insulin topical gel significantly has better outcome than short acting insulin topical gel, simvastatin $2 \%$ topical gel, simvastatin 3\% topical gel and control group. The number of fibroblasts in long acting insulin topical gel is higher than

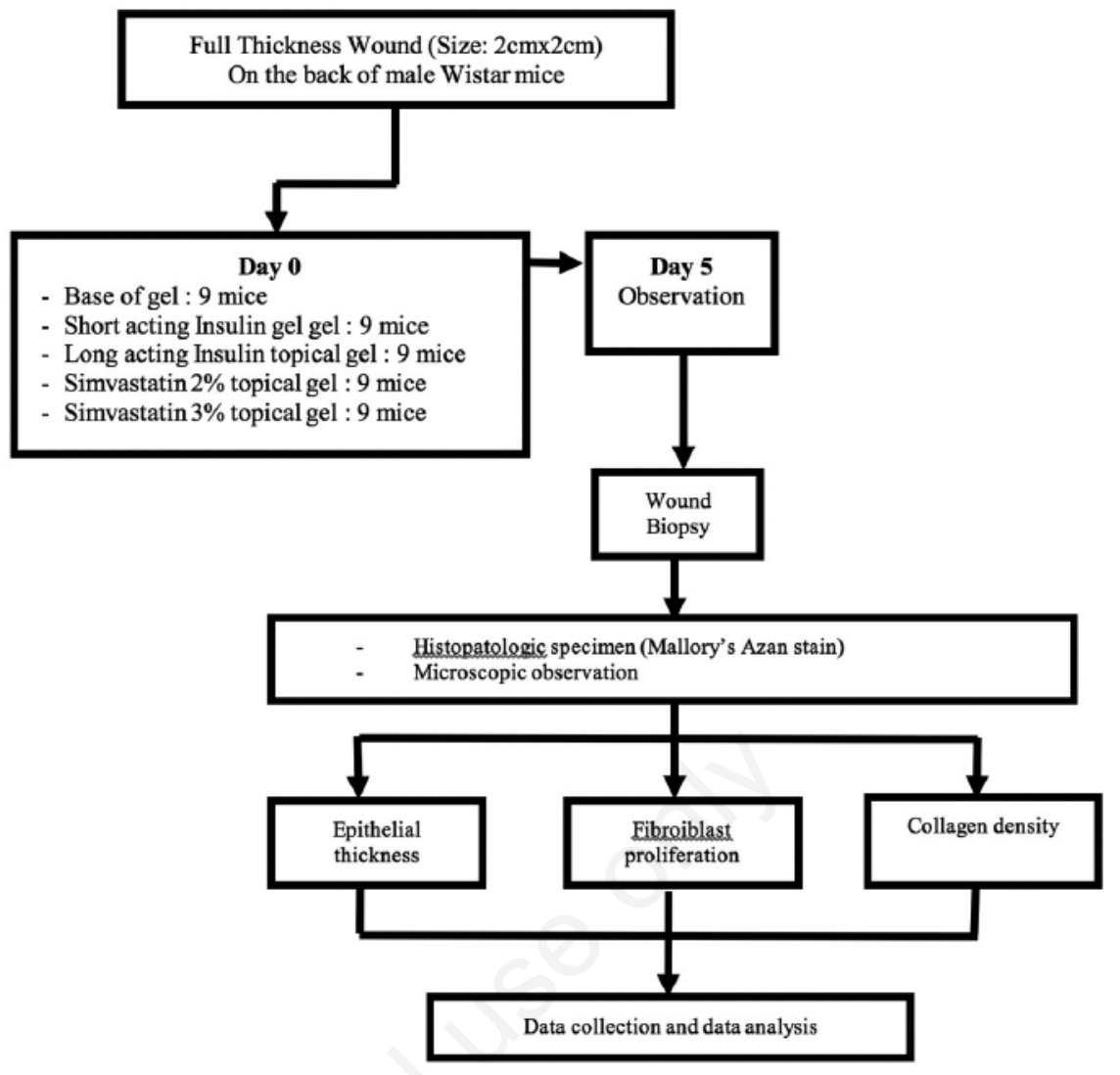

Figure 1. Experimental study scheme.

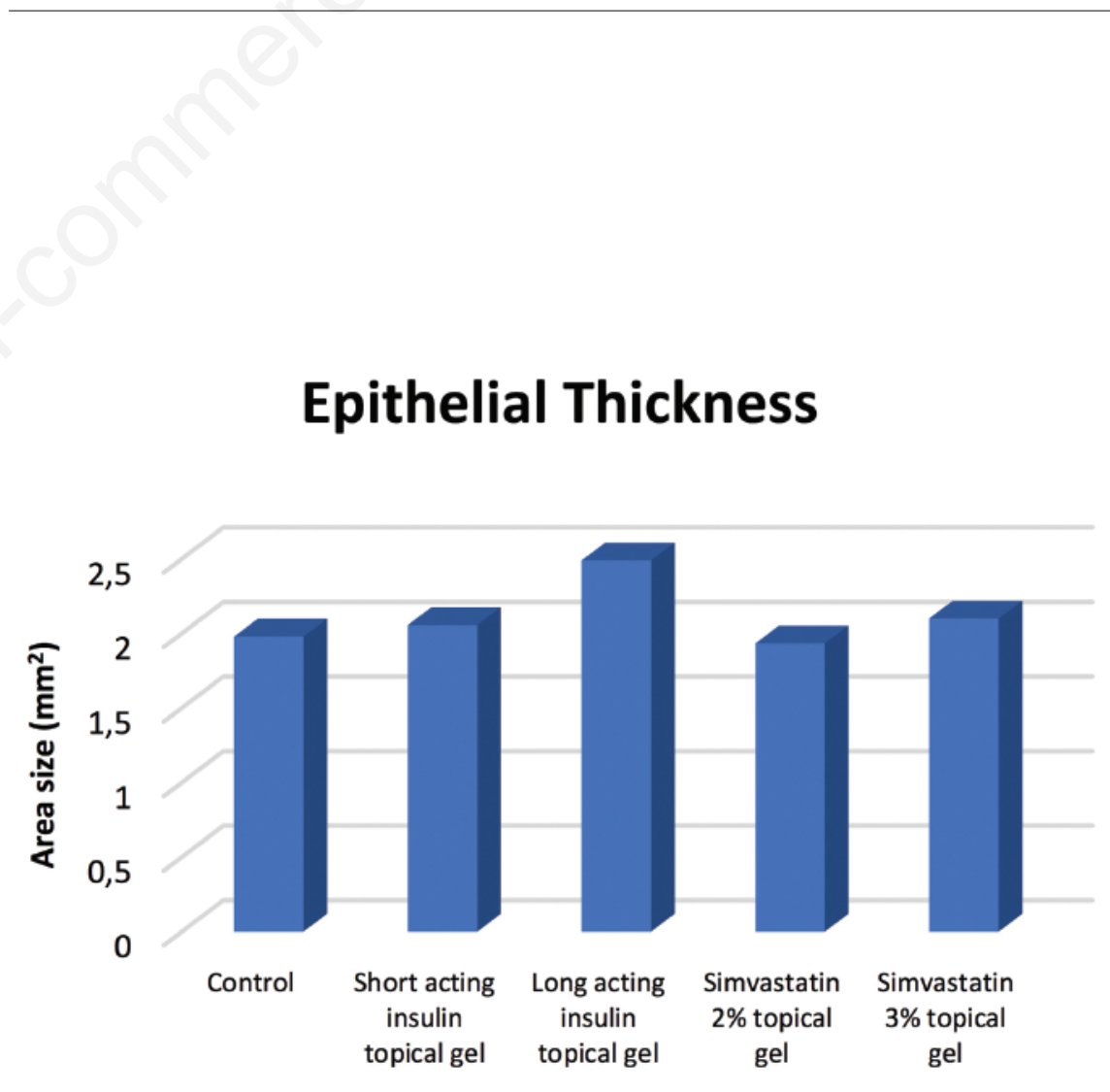

Figure 2. Epithelial Thickness after day 5 of study. 
Fibroblast Proliferation

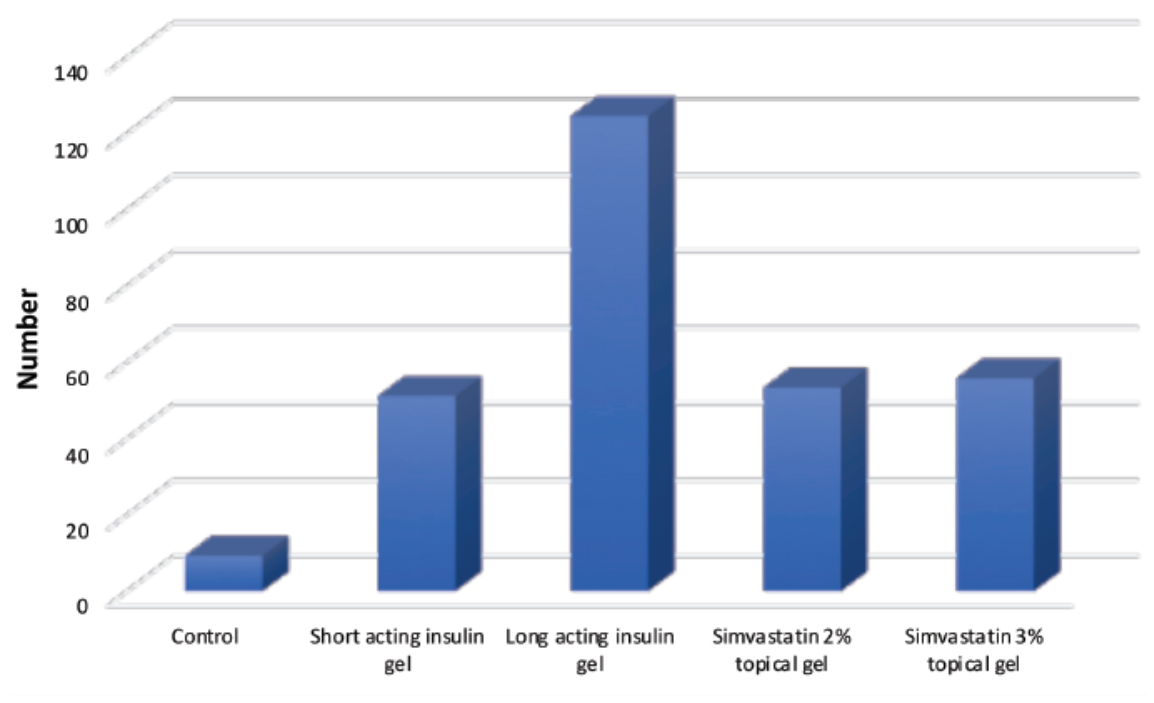

Figure 3. Fibroblast proliferation after day 5 of study.

\section{Collagen Density}

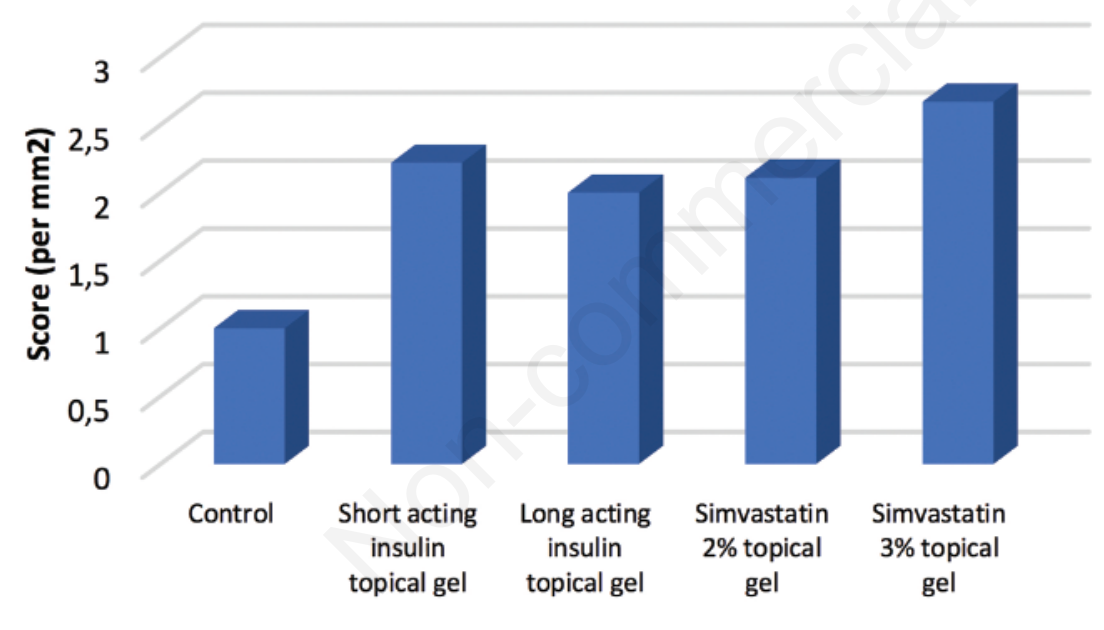

Figure 4. Collagen density after day 5 of study. other group and it has a significant different number from other groups. On the other hand, the level of collagen density in simvastatin 3\% gel is significantly higher than the other group.

Further studies are needed to support this study such as a study with more parameters and longer period of time to analyze the biomolecular effectivity of insulin topical gel and simvastatin topical gel; moreover, a human study that analyze the short and long term effect of insulin topical gel and simvastatin topical gel on wound in normal blood glucose level and diabetic patients.

\section{References}

1. Stojadinovic O, Lebrun E, Pastar I, et al. Statins as potential therapeutic agents for healing disorders. Expert Rev Dermatol 2010;5(6);689-98.

2. Khoshneviszadeh M, Ashkani-Esfahani $\mathrm{S}$, Namazi MR, et al. Topical simvastatin enhances tissue regeneration in full-thickness skin wounds in rat models. Iran J Pharm Res 2014;13(1);263-9.

3. Buchanan EP, Longaker MT, Lorenz HP. Fetal skin wound healing. Adv Clin Chem 2009;48;137-61

4. Thorne C, Chung KC, Gosain A, et al. Wound healing: normal and abnormal. In: Thorne $\mathrm{CH}$, Chung $\mathrm{KC}$, eds. Grabb and Smith's plastic surgery. 7th ed. Philadelphia: Lippincott Williams \& Wilkins; 2014.

5. Asai J, Takenaka H, Hirakawa S, et al. Topical simvastatin accelerates wound healing in diabetes by enhancing angiogenesis and lymphangiogenesis. Am J Pathol 2012;181(6);2217-24.

6. Apikoglu-Rabus S, Izzettin FV, Turan P, Ercan F. Effect of topical insulin on cutaneous wound healing in rats with or without acute diabetes. Clin Exp Dermatol 2010;35(2);180-5. 